\title{
Wind inhomogeneities in [WC] central stars of planetary nebulae
}

\author{
Yves Grosdidier \\ Ernest Rutherford Physics Building, McGill University, \\ 3600 rue University, Montréal, QC H3A 2T8, Canada
}

Anthony F.J. Moffat

Département de physique, Université de Montréal, C.P. 6128, succ. Centre-Ville, Montréal, QC H3C 3J7, Canada, and Observatoire du mont Mégantic, Canada

Agnès Acker

Observatoire Astronomique de Strasbourg, UMR 7550, 11 rue de l'Université, F-67000 Strasbourg, la France

In the present poster paper, we summarize the observational spectroscopic evidence for wind clumping originating in some [WC8-10] and [WO4] central stars of planetary nebulae. Specifically, we concentrate on the CiII $\lambda 5696$ and CIV $\lambda \lambda 5801 / 12$ emission-line variability observed for well-known planetary nebula nuclei. The main results are:

(i) All the [WC]-late stars considered so far (HD 826, BD+30 $3639, \mathrm{He} 2-99$, He2-142, SwSt 1) show significant or marginal (SwSt 1 and He2-142) line profile variations of their C III $\lambda 5696$ emission line, the best line to show this effect (Grosdidier et al. 2000, 2001). For HD 826 and $\mathrm{BD}+30^{\circ} 3639$, all the stellar emission lines within the $5500-6000 \AA$ spectral range are variable. The amplitudes of the variations range from about $5 \%$ up to $25-30 \%$ of the adjacent continuum flux, over timescales of hours (Grosdidier et al. 2000, 2001).

(ii) New results discussing the case of the hotter subtype [WO4] (Grosdidier \& Acker, in preparation; Acker \& Grosdidier 2002) show that the variability observed in the CIV line of NGC 1501 and NGC 6751 ranges up to $10 \%$ of the adjacent continuum flux, over timescales of hours or days. This result is essentially the same as found for [WC]-late type stars.

(iii) The subpeaks normally show measurable velocity shifts in HD 826 and $\mathrm{BD}+30^{\circ} 3639$ during their lifetime. Subpeaks/gaps on the top of the CIII line generally move from line center towards line edges in a symmetric fashion in the blue and red edges (Grosdidier et al. 2000, 2001). The lifetime of the subpeaks is roughly a few hours. Therefore, these winds are highly variable on a very short time-scale. 
(iv) The kinematics of moving structures on top of the C III $\lambda 5696$ line of HD 826 and $\mathrm{BD}+30^{\circ} 3639$ have been measured and compared to similar studies on massive WRs. At least in the case of HD 826 (and possibly most of the [WC] stars), the maximum acceleration is significantly larger than that found for its massive spectroscopic counterpart, WR 135 . This is attributed to the very small radius of HD 826 (Grosdidier et al. 2001, 2002).

$(v)$ The line variability of late-type [WC] nuclei is very similar to that observed in the massive Wolf-Rayet stars WR 103 (WC9) or WR 135 (WC8); see Robert (1992), Lépine \& Moffat (1999), and Lépine et al. (2000). Thus, the wind fragmentation process appears to be a purely atmospheric wind phenomenon, despite the strong differences between both types of underlying hot stars.

(vi) On the whole, the winds of all [WC] central stars appear significantly stochastically variable on relatively short time-scales. This supports a turbulent origin.

(vii) We expect strong, hydrogen-deficient [WC] winds to be extreme examples for central stars of PN, so that any fine structure found in [WC] winds may apply to all winds of central stars of PN, much as one is finding now that weak, massive O-star winds also show the same fine structure as massive WR winds.

(viii) The consequences of clumping in hot-star winds are manifold, including substantial constraints on the effective mass-loss rates, and their possible impact on the surrounding nebula itself. Recall Acker et al. (2002) who found systematic turbulent velocities originating in PNe around [WC] stars, while the nebulae around O-type central stars show no clear evidence for turbulent motions. Although stellar wind clumps cannot reach large nebular distances easily, they can play an important role during the momentum-driven phase, which is known to be very sensitive to the non-linear thin-shell instability, as well as to other hydrodynamical instabilities. Therefore, the turbulence detected in surrounding nebulae could be either triggered, or enhanced, by stellar wind clumps that appear ubiquitous in Population-I and -II WR stellar winds.

$(i x)$ Finally, the extended distribution of spectral types among WR central stars may provide a broad baseline for comparison and detection of overall trends that otherwise might be drowned out in data generally showing large intrinsic dispersions within a given WR spectral type.

\section{References}

Acker, A., Gesicki, K., Grosdidier, Y., Durand, S. 2002, A\&A 384, 620

Acker, A., Grosdidier, Y. 2002, in: M.A. Dopita, S. Kwok \& R.S. Sutherland (eds.) Planetary nebulae: their Evolution and Role in the Universe, Proc. IAU Symp. No. 209, in press

Grosdidier, Y., Acker, A., Moffat, A.F.J. 2000, A\&A 364, 597

Grosdidier, Y., Acker, A., Moffat, A.F.J. 2001, A\&A 370, 513

Grosdidier, Y., Acker, A., Moffat, A.F.J. 2002, RMxAA-SC 12, 90

Lépine, S., Moffat, A.F.J., St-Louis, N., et al. 2000, AJ 120, 3201

Lépine, S., Moffat, A.F.J. 1999, ApJ 514, 909

Robert, C. 1992, PhD thesis, Université de Montréal, Canada 